\title{
A Conceptual Note on Gesture Theory
}

\author{
*Juan Sebastián Arias-Valero \\ Universidad Nacional Autónoma de México \\ jsariasv1@gmail.com \\ Orcid: 0000-0001-6812-7639 \\ EMilio Lluis-Puebla \\ Universidad Nacional Autónoma de México \\ lluisp@unam.mx \\ Orcid: 0000-0002-2633-5955 \\ DOI: $10.46926 /$ musmat.2021v5n1.89-115
}

\begin{abstract}
This paper is a conceptual counterpart of the technical developments of gesture theory. We base all our discussion on Saint-Victor's definition of gesture. First, we unfold it to a philosophical reflection that could establish a dialogue between semiotic and pre-semiotic approaches to musical gestures, thanks to Peirce's ideas. Then we explain how the philosophical definition becomes a mathematical one, and provide reflections on important concepts involved in gesture theory, some possible relations to other branches of mathematics and mathematical music theory, and several open and closed questions. We include a non-mathematical discussion related to the environment of this subject and a glossary of specialized terms.
\end{abstract}

Keywords: Gestures. Mathematical Music Theory. Category Theory. Geometry. Philosophy.

\section{TO THE READER}

I he following lines try to explain some concepts in gesture theory by means of general ideas, motivations, and musical examples. We do not pretend that the reader understands all mathematical details. Regarding the technical concepts that we consider as essential to understand the big picture, we include a glossary, to which the reader will be directed after clicking on a red term, like topological gesture. However, it is desirable that the reader has a minimum acquaintance with category theory [18, pp. 10-23]. On the other hand, we end each example with the symbol $\boldsymbol{\alpha}$ for organization.

*This work was supported by Programa de Becas Posdoctorales en la UNAM 2019, which is coordinated by Dirección de Asuntos del Personal Académico (DGAPA) at Universidad Nacional Autónoma de México.

Received: February 26th, 2021 Approved: April 21th, 2021 


\section{SOME MOTIVATIONS AND ANTECEDENTS}

The first version of The Topos of Music [28] was an enterprise that achieved a framework for musicology based on topos theory, including a theory of performance and a very complete account of the mathematical structures present in music. Soon after this accomplishment, Mazzola became aware that his own activity as a free jazz pianist had little to do with the structures and procedures described in his monograph; see [23, Chapter 24]. Gestures, rather than formulas, were the essence of his performance. Certainly, free jazz improvisation is mainly determined by the movements of the body's limbs, that is, by a dancing of the body, the classical structures of Western music being secondary and auxiliary. Therefore, a rigorous reflection on gestures was necessary, and not only in the case of musical improvisation, but in music in general, since all its power and intensity relies in its realization in bodily terms, even in the Western classical tradition.

Indeed, the task of studying gestures in music is not new and has received considerable attention from other musicologists in recent times, though following different approaches to formulate a suitable conceptualization of musical gestures. In fact, these studies are far from a unified treatment of the concept of gesture. On the one hand, some authors [13, Chapter 1] emphasize the need of content, meaning, and signification in the definition of gesture arguing that the understanding of a gesture as a mere movement of the body can lead to an ambiguity regarding whether this movement expresses a musical intention or not, like in the case of the gesture of a listener in response to music [13, § 1.2.4]. On the other hand, studies like [10] have an inclination for more flexible definitions of gesture, in which the notion of meaning or signification need not be included:

"We consider that the word gesture (or the French equivalent geste) necessarily makes reference to a human being and to its body behaviour-whether they be useful or not, significant or meaningless, expressive or inexpressive, conscious or not, intentional or automatic/reflex, completely controlled or not, applied or not to a physical object, effective or ineffective, or suggested." [10, p. 73]

However, most of definitions seem to have a common point: a gesture is the movement of the body (plus several nuances).

\section{SAINT-VICTOR'S DEFINITION AND ITS PRAGMATICIST DEVELOPMENT}

The point of departure for all, both formal and informal, developments discussed in this paper, is the philosophical definition of gesture given by Hugues de Saint-Victor in the chapter XII of [11]: "Gesture is the movement and configuration of the body's limbs, towards an action and having a modality."1

In Section III, we comment in detail how it is possible to translate this definition into mathematical terms, but before we explore its pragmaticist essence, as pointed out in [37]. More precisely, we will apply Peirce's phaneroscopy and see how a semeiotic framework, which is not presupposed in the definition but a consequence of certain particularities of Western music, naturally emerges after triadic elaboration in the phaneron. However, this does not mean that all gradations of the definition in the phaneron are semiotic.

\footnotetext{
${ }^{1}$ Our translation of the original quote: "Gestus est motus et figuratio membrorum corporis, ad omnen agendi et habendi modum."
} 


\section{i. Peirce's phaneroscopy}

According to Peirce, phaneroscopy, or phenomenology, is the study or description of the phaneron defined as the complete collective that is present to the mind; see [36, Chapter 3] and [33, p. 74]. Phaneroscopy includes the study of the cenopythagorean categories, the three modes of being, or the tints occurring in phenomena. We use the synthesis of the three categories made in [36, Chapter 3], which relates these to keywords as follows:

Firstness: immediacy, first impression, freshness, sensation, unary predicate, monad, chance, possibility.

Secondness: action-reaction, effect, resistance, alterity, binary relation.

Thirdness: mediation, order, law, continuity, knowledge, ternary relation, triad, generality, necessity.

In what follows, we regard these categories as the three fundamental modes from which we progressively stratify Saint-Victor's definition, and use them by recursion.

\section{ii. Movement and aim}

The fundamental observation of Fernando Zalamea, in the introduction to [37], that Saint-Victor's definition of gesture as "the movement and configuration of the body's limbs with an aim" is fully pragmaticist in Peirce's sense, can initially be interpreted as the fact that it is related to thirdness. If a gesture is the movement and configuration of the body with an aim, then it is by definition a mediation between two states of the body; a former state or beginning, and a second state or aim. In turn, the movement implies continuity and it is an essential manifestation of thirdness, so $a$ gesture is marked by thirdness.

As the beginning of a gesture, a first state of the body is firstness. As the end of a gesture, the aim is secondness. The aim necessarily refers to a first state of the body, so it has a dyadic character. According to [33, p. 76]: "The beginning is first, the end second, the middle third. The end is second, the means third. The thread of life is a third; the fate that snips it, its second." The modalities of this thirdness will lead to semeiotics.

\section{iii. Modality}

Now we add to our discussion a new term from Saint-Victor's definition, namely modality, which is a new trace of pragmaticism. We may relate it to the three categories of Peirce again to give modal sub-determinations of gestures.

For simplicity, let us consider the instance of piano performance. There is a certain tension between the elasticity of the performer's body and the stability of the instrument, which is mediated by the gesture of the interpreter. Three initial modes of the gesture regarding the piano can be considered.

1. A raw movement, potential but not reactive. Not all movements of the performer produce an effective touch of the piano, and hence sounds. Precisely, we refer to those auxiliary gestures in a state of firstness that suggest moods, directions, waves, or continuities, and that are spontaneous and immediate. They are a bodily envelope for sound. Here we recall the famous hums and envelopes of Glenn Gould and Keith Jarrett.

2. The movement that effectively acts on the instrument and produces sounds. This is the activereactive movement of the body. Besides touch, or the active character of the movement, and a first reaction, or the feel of the instrument, there is the main reaction of that touch of the instrument: sound. When the body touches the instrument, it vibrates and this vibration, when propagates 


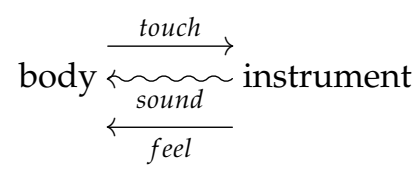

Figure 1: Diagram describing the active-reactive movement of the body.

through the thickness of air and space, is the sound itself. We picture this situation in the diagram of Figure 1. Here, we remember the violent chords from Bartók's Allegro Barbaro or Scriabin's Study Op. 8 No. 12.

3. Coordination-performance: the movement mediates between the touch of the instrument and the sounds that are produced. A first mediating instance is hearing, or the reactive action of the sound on the body with a certain degree of consciousness. ${ }^{2}$ The body is embedded in sound and is enveloped by it, but it reverberates inside the body, makes it vibrate, and, in particular, it affects the brain. Hearing and feeling the sound and the instrument are first instances of interaction and communication in the performance, and allow the performers to take their own decisions about how their movement should become so as to have the appropriate modality when coordinating touch and sound. These modalities are the particularities of each performer; their modes of being regarding the instrument. This opens up a new opportunity to apply the three Peircean modes.

\section{iv. Natural emergence of semeiotics}

In a vague way, three further subdeterminations of coordination-performance can be distinguished, according to the Peircean categories. In this case, modality is introduced in the way that the sound is produced: a spontaneous one, one given by the opposition between forces, or one given by a law or design.

3.1. Free improvisation. Probably related to a strong back-and-forth between conscious and subconscious states of the body-mind, free improvisation is spontaneous and is hardly based on previous references or on limitations of musical resources (space, techniques, instrumentation). This is fully exemplified by Mazzola's vision of his activity as a free jazz pianist as one related to immediate gestures rather than mediating formulas. As in the mathematical notion of free object [17, Section III.1], in free improvisation the structure is not totally absent, but is skeletal. According to [22, p. 7], free jazz musicians generate their music partaking in a dynamic and sophisticated game, whose rules are incessantly generated and/or recycled, not prescribed, and negotiating what they are going to play. The skeletal configuration, related to these rules, allows a high capacity of transformation and internal movement (free objects have special properties of projection on the objects of the same category), which tends to multiplicity and freshness.

3.2. Thematic improvisation. Related to the dialectics facts/concepts and visible/non-visible, it is the improvisation that consciously uses a determined system of musical techniques or concepts. If we are located within jazz standards and academic traditions of music, we can define improvisation as the live (while playing) creation with a wide knowledge of musical techniques (harmony, counterpoint, composition).

3.3. Mediated interpretation. Perhaps the most important mediation in music performance is

\footnotetext{
${ }^{2}$ Following Merleau-Ponty's late ideas [30], this definition is insufficient since perception cannot be reduced to the action of the perceived on the perceiver. Moreover, the introduction of consciousness is also problematic since its definition is far from being clear. However, this first approximation is useful for relating musical gestures and Peirce's ideas.
} 


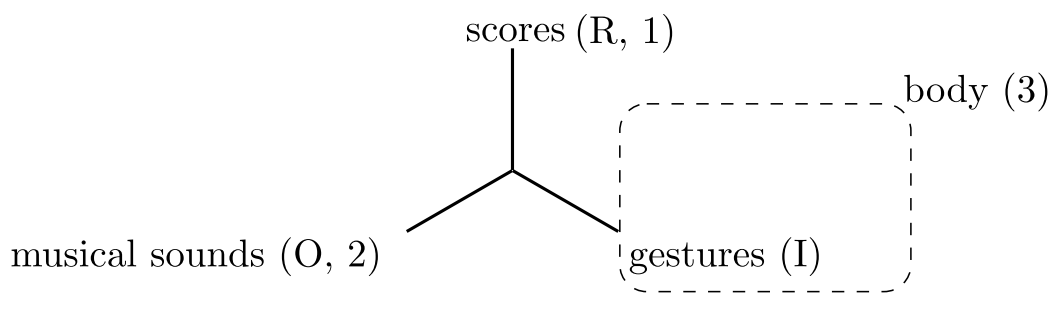

Figure 2: The triad of Western performance as a Peirce's sign.

vision, whether actual or as an image in the mind. Interpretation, in traditional sense, takes place according to the design of a score. At this point of considerable triadic elaboration, the fundamental sign of the following discussion emerges in a natural way. The sign is Mazzola's triad sounds/scores/gestures of Western musical performance, which we study in the following section.

\section{v. Semeiotics and Mazzola's triad of Western musical performance}

According to Peirce, knowledge occurs through signs. ${ }^{3}$ A sign, in Peirce's sense, consists of an object $\mathrm{O}$ (or second 2), a representamen $\mathrm{R}$ (or first 1 ), and a quasi-mind (or third 3 ), such that the representamen replaces the object for the quasi-mind, in which the representamen is transformed into the interpretant I of the sign. The quasi-mind need not be related to a human mind; rather it corresponds to the interpretation context where the semeiosis, that is, the transformation of the representamen into the interpretant, occurs. Similarly, the terms O, R, and I are quite flexible: they can be things, concepts or even signs, giving place to endless semeiosis, which refers to the infinite iteration of signs.

The sign sounds/scores/gestures of Western musical performance, introduced in Section iv, can initially be schematized by means of Figure 2. There, $\mathrm{O}$ is musical sounds, $\mathrm{R}$ is scores, and we regard the body as the quasi-mind (3) where the scores are transformed into gestures (I).

The exhibition of the sign is only the point of departure for the process of endless semeiosis. Certainly, formulas and gestures are different representations of sounds, ${ }^{4}$ and more dramatically, the terms $\mathrm{O}, \mathrm{R}$, and I are transmutations of each other: scores produce gestures in the body, which produce new sounds by means of the interaction with an instrument, which in turn produce scores in an appropriate (quasi-)mind (think of the process of transcription of a musical piece, or some process of codification of sound), and so on, in an endless spiral. Within this logic of endless semeiosis, there are more possibilities. First, the musical sounds considered can also be representations of previous concepts; in fact, musical ideas can be related to previous ones or even to non-musical ideas like those of poetry or some kind of text. Also, scores can be the interpretant of previous ideas that represent musical sounds; for example, motives, or the subjects on which fugues are developed.

\footnotetext{
${ }^{3}$ According to one of the reviewers: "This is exactly what Mazzola's gesture theory questions, and this is also congruent with the approach of important French diagrammatic philosophers, such as Alunni and Châtelet." However, we do not see a marked opposition of these philosophers with the more general Peirce's phaneroscopy (Section i), since both knowledge and gesture are thirdness, and hence are strongly related. Thus, in the same way as we located gestures (Section ii) and musical semeiotics (Section iv, Paragraph 3.3) in the phaneron, we might pursue French gestural ideas (also including Merleau-Ponty, Cavaillès, and Desanti).

${ }^{4}$ Although the main point of musical gesture theory is that gestures generate sounds, they can also be representations of sounds, like a dancer's gestures in response to music.
} 


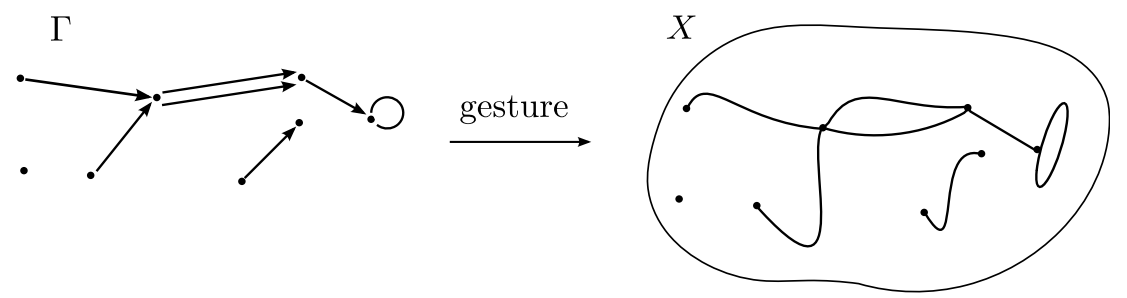

Figure 3: A gesture in a topological space $X$ shaped by a digraph $\Gamma$.

\section{THE HYPERGESTURAL APPROACH}

Saint-Victor's definition ${ }^{5}$ is also the inspiration for mathematical gesture theory, as discussed in what follows. In this section, we present Mazzola's initial mathematical insights.

\section{i. Configuration, body, and space}

Based on Saint-Victor's definition, Mazzola and Andreatta give the first mathematical definition ${ }^{6}$ of gesture, namely that of topological gesture, as a diagram of curves in a topological space $X$; see Figure 3. The shape of the gesture is given by a digraph $\Gamma$, which consists of arrows and vertices suitably connected, and the diagram incarnates the arrows as continuous paths in $X$ and the vertices as points in the space, preserving the configuration of $\Gamma$.

In terms of Saint-Victor's definition, the digraph corresponds to the configuration of the body's limbs and the topological space corresponds to the space-time where the movement of the body occurs. The absence of a load of significance is deliberate in this definition and corresponds to Mazzola's presemiotic approach. A gesture has an existence in its own right, regardless of whether or not it conveys any meaning, as in the cases of dancing or free improvisation. However, as we observed before (Section iv, Paragraph 3.3), the mediation of scores in performance leads to semeiotics.

Example 1. We can define gestures in musically meaningful spaces. Let us interpret the Euclidean space $\mathbb{R}^{2}$ as the score space, where a pair $(t, p)$ denotes a sound event with pitch $p$ that occurs at the time $t$. We choose the unities according to the situation. In the following discussion we use the quarter duration as time unity and identify the subset $\mathbb{Z}$ of $\mathbb{R}$ with the diatonic scale indicated by the key involved. For instance, the pair $(1 / 2,0)$ denotes the pitch A4 occurring after an eight duration.

Consider the first phrase of Mozart's Piano Sonata ${ }^{7}$ K. 331. The melodic contour can be regarded as a gesture in $\mathbb{R}^{2}$, see Figure 4 . As we explain in Examples 4 and 5, the melodic contour plays an important role in Mozart's variations of this phrase since they can be regarded as the result of transforming the original gesture with homotopies and sheaves.

\footnotetext{
${ }^{5}$ This definition was used by Jean-Claude Schmitt in [35] before the mathematical definition in Section i was formulated by Mazzola and Andreatta.

${ }^{6}$ This definition appeared for the first time in [27]. Other detailed presentations can be found in [3, Section 1.1] and [4].

${ }^{7}$ The authors prepared the excerpts from [32] (theme and variation 1) and the original manuscript (variation 4) at https://mozart.oszk.hu/.
} 


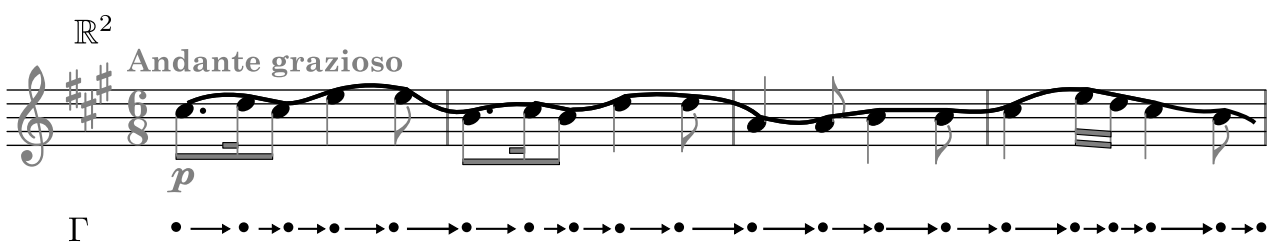

Figure 4: The first phrase in Mozart's K. 331 as a gesture.

\section{ii. Hypergestures}

Nevertheless, it is not clear why this definition models the human body. For example, the contour gesture in Figure 4 is bi-dimensional. Mazzola's solution to this problem consists in constructing hypergestures, which are just gestures of gestures. If we want a gesture of gestures, then our definition requires the construction of a space of gestures replacing $X$. This construction is one of the central concepts of gesture theory. In fact, we can equip the set of all $\Gamma$-gestures in $X$ with a natural topology, in the sense that it is a categorical construction with a universal property. Thus, once we have the space of gestures $\Gamma @ X$, given another digraph $\Gamma^{\prime}$, we can consider $\Gamma^{\prime}$-gestures in $\Gamma @ X$ and the space of hypergestures $\Gamma^{\prime} @ \Gamma @ X$. This construction leads to models of the human body. ${ }^{8}$

\section{Gestures AND the NOtion OF SPACE}

Traditionally, we model space-time with the Euclidean space $\mathbb{R}^{n}$, for instance, in classical mechanics. However, it is worth to ask whether space and body, which is embedded in space, are decomposable in terms of points, like $\mathbb{R}^{n}$ or any topological space.

\section{i. Locales}

Locales are generalized topological spaces ${ }^{9}$ whose primitive notion is that of open, regardless of whether or not the latter has points. This is more congruent with the fact that we tend to experience space and time in terms of neighborhoods and intervals, and that our bodies are more the result of the synergy of organs and limbs rather than aggregates of atoms.

Since locales need not have points, which are possible incarnations of the vertices of a digraph, it is not reasonable to define an individual gesture in a locale. Instead, we consider locales of gestures that are analogous to spaces of gestures, and can be computed via the categorical constructions of limits and exponentials. There are non-trivial locales of gestures with no points at all, as shown in the following example.

Example 2. In contrast to the idealized model in Example 1, a musical sound cannot exist in a time instant -instead it is a vibration that needs an interval of time to propagate. Consider the topology on $\mathbb{R}$ whose basic opens are all half-closed intervals of the forms $[a, b)$ and denote the associated topological space by $H$, which is Hausdorff. We interpret these intervals as those time spans where a sound usually exists.

\footnotetext{
${ }^{8}$ Drawings of approximations to the human body by means of hypergestures are in [27, p. 33]. We do not include similar figures here since, in Section $\mathrm{V}$, we present an alternative and more natural model of the human body based on simplicial sets.

${ }^{9}$ In fact, the wide class of sober spaces, which includes Hausdorff and have the property that we do not loose information by replacing them by their lattices of opens, is embedded in the category of locales.
} 


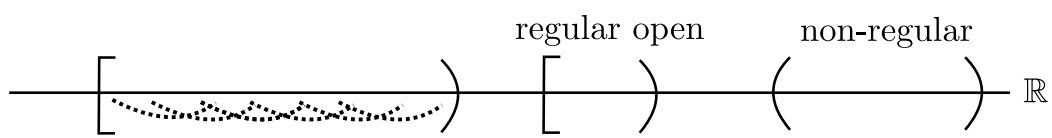

element in the locale of gestures

Figure 5: An element of the locale of all opens in $H$ whose closures coincide with their interiors, also called regular opens (center), which can interpreted as time spans with onset where sound can exist. A non-regular open (right-hand side). An element in a typical locale of gestures (paths), which consists of an indecomposable family of gestures, depicted as pointed curves (left-hand side).

We consider the locale $L$ of all opens in $H$ coinciding with the interior of their closures, where the join and meet is given by the interior of the closure of the union and intersection in $H$, respectively. In this case, half-closed intervals are in $L$, whereas open intervals of the form $(a, b)$, which have no onset, are not-see Figure 5. Also, this locale is an example of locale with no points at all ${ }^{10}$ and can be regarded as a non-atomic model of time reconstructed from the existence of sound.

On the other hand, we can consider the exponential locale of paths $L^{\mathcal{O}(I)}$, which is just the locale of gestures on $L$ for the arrow digraph $\bullet \rightarrow \bullet$. It could be interpreted as a locale of gestures on time, induced by gestures on space-time. This locale has no points again, ${ }^{11}$ but has many elements representing indecomposable families of gestures. Indeed, the locale $L^{\mathcal{O}(I)}$ is generated [16, p. 320] by the symbols of the form $W(u, a)$, where $u \in \mathcal{O}(I)$ and $a \in L$, which represent families of paths, akin to the generators of a compact-open topology. For example, $W(I, a)$ represents the family of paths whose image is contained in $a$; see Figure 5.

The fact that locales need not have points and their limits and exponentials are difficult to compute could be unattractive. Certainly, we are used to thinking analytically, in terms of points, but it is a profound question whether locales are more appropriate to model space-time and the human body than topological spaces.

\section{ii. Grothendieck topoi}

Nevertheless, locales are a bridge between topological spaces and the broader notion of space, namely Grothendieck topoi, which is central in mathematics. Grothendieck topologies are defined in categories and their primitive notion is that of covering, instead of those of point or neighborhood. In a sort of yoga, Grothendieck replaces a site (category with a Grothendieck topology) by its category of sheaves, the latter called Grothendieck topos, which has all limits and colimits, in much the same way as a topology is suitably closed under intersections and unions. We have accessed the continuous realm from the discrete in an astonishing way. ${ }^{12}$

The potential of recovering the substantial movement behind functions and, more generally, morphisms, was one of the essential features of gestures stressed by Mazzola in his first papers on gestures [27, 24]. For example, the linear transformation of the plane associated with a rotation matrix only takes into account the beginning and end but not the intermediate movement, which is a gesture. This gave rise to the question whether there is an analogue of the Yoneda embedding that allows to represent a given category in terms of gestures. The Yoneda embedding naturally

\footnotetext{
${ }^{10}$ See [18, p. 489]. An alternative proof can follow the lines of [3, p. 20].

${ }^{11} \mathrm{In}$ fact, there is a homeomorphism [3, Proposition 2.4.2] $p t(\Gamma @ L) \cong \Gamma @ p t(L)$ and $p t(L)=\varnothing$, so $p t(\Gamma @ L)=\varnothing$ whenever $\Gamma$ has at least a vertex, where $p t$ assigns to each locale its space of points.

${ }^{12} \mathrm{~A}$ complementary discussion on the emergence of Grothendieck topologies and their relation to sheaves can be found in Section VI.
} 


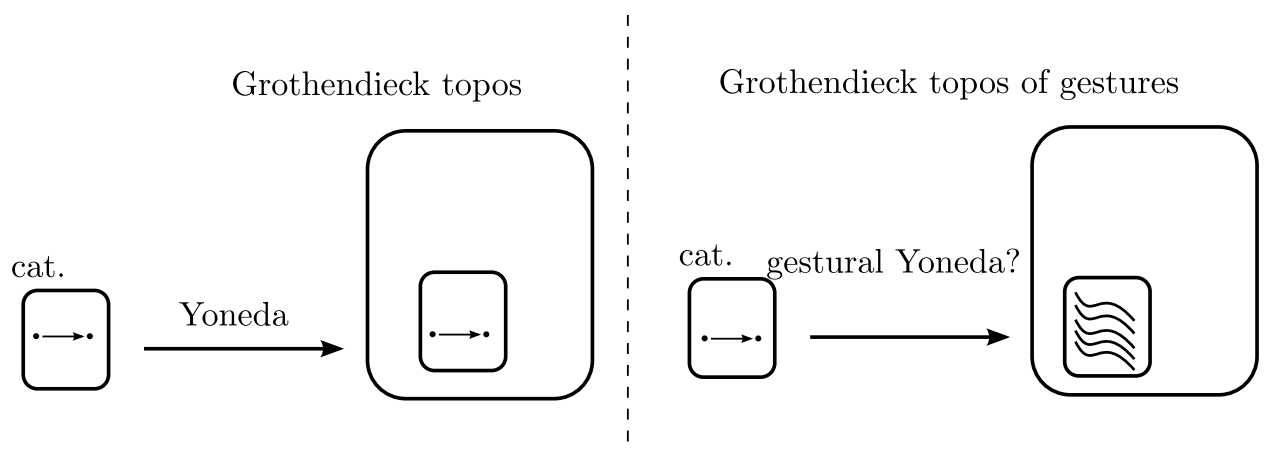

Figure 6: The Yoneda embedding in a Grothendieck topos of presheaves (left-hand side) and a possible gestural Yoneda embedding (right-hand side).

represents a given category as a full subcategory of its associated category of presheaves, the latter being a particular example of Grothendieck topos, since presheaves are sheaves for a certain trivial Grothendieck topology. This means that the Yoneda embedding is already a representation in a generalized space, so a possible gestural embedding could be refined thereof; see Figure 6 .

For this reason, some authors embarked on the enterprise of defining gestures on Grothendieck topoi [3, Chapter 4]. Once again, there can be no points, so, as locales taught us, the definition of a gesture is not a reasonable approach. Instead, we define a Grothendieck topos of gestures, but this definition has an important difference. The natural structure of the collection of all Grothendieck topoi is not that of a category, but it is a 2-category, ${ }_{1}^{13}$ which also takes into account the natural transformations between geometric morphisms (the analogues of continuous maps) of Grothendieck topoi. Besides, the notion of limit, which is basic for constructing an object of gestures, is not appropriate in this context, and rather we talk about bilimits. Consequently, we define the Grothendieck topos of gestures as an appropriate bilimit. This renders the theory of gestures on Grothendieck topoi very challenging in technical terms. For example, for profound reasons, we can only ensure the existence of the Grothendieck topos of gestures if the digraph involved is finite [3, Remark 4.2.3].

Up to now, we do not know whether this notion effectively helps solve the problem of the gestural Yoneda embedding. However, the consideration of this level of generality was essential in the development of a theory of gestures [3]. First, it led to consider the intermediate case of locales, which is halfway between Grothendick topoi and topological spaces and concentrates the spatiality of Grothendieck topoi. Second, it was useful for formulating a comprehensive definition of gestures on objects of categories, by means of limits, and on objects of 2-categories, by means of bilimits, without using points.

Next, we discuss Mazzola's approach to the gestural Yoneda embedding problem.

\section{iii. Topological categories}

Another way of introducing topologies in categories corresponds to topological categories. A topological category is a category such that their sets of objects and morphisms are topological

\footnotetext{
${ }^{13}$ Unlike other concepts in the Glossary, providing complete definitions of 2-category [17, Section XII.3], bilimit, and exponential in 2-categories, which would take tens of pages, will not improve the intuition of the non-specialists on the subject. It is enough to bear in mind the analogies topological space/Grothendieck topos, continuous function/geometric morphism, limit/bilimit, and topological space of gestures/Grothendieck topos of gestures. We only include the discussion to motivate the concepts and invite the reader to review [3, Section 4.2].
} 
spaces and all operations (domain, codomain, identity, composition) involved in the category definition are continuous. Thus, topological categories mix point-set topology and categories.

Example 3. The score space in Example 1 can be regarded as a topological category. The objects are the elements $x \in \mathbb{R}^{2}$, and the unique morphism from $x$ to $y$ is the triple $(x, y, y-x)$, where $y-x$ is the vector difference. The third component $y-x$ is the interval between the sound events $x$ and $y$. For example, the third component $(1 / 2,-2)$ denotes a descending third that occurs between two sound events separated by an eight time interval. The composition of two such interval morphisms is given by the formula in Equation 1, and it corresponds to addition in the intervallic components:

$$
(y, z, z-y) \circ(x, y, y-x)=(x, z, z-x),
$$

The topological space of objects is $\mathbb{R}^{2}$ with the usual topology, and the space of morphisms is a subspace of $\mathbb{R}^{2} \times \mathbb{R}^{2} \times \mathbb{R}^{2}$ with the product topology. This category is actually a groupoid since its morphisms are invertible, and mix the algebraic intervallic and topological structures of the score space.

The paper [24] proposed the existence of a bicategory of gestures, based on topological categories, as a possible universe for the Yoneda embedding. However, the proposed embedding was not full. We comment another proposed solution by Mazzola in Section VI.

Based on the original sketches [24], the complete definition of gestures on topological categories is in [1] and [6], together with some additional developments of the theory. Regarding the definition, the last paper studies the explicit topological category structure of the topological category of gestures, including constructive examples-the explicit presentation of the morphisms and topologies on morphisms and objects of the topological category of gestures were absent in [24, Section 2.2]. On the other hand, the exponential presentation problem of a given topological category of gestures, which relates it to a category of functors with domain the category of continuous paths of a digraph, was initially addressed, but not solved, in [6]. This includes a complete description of the topological structure of the mentioned category of continuous paths, which is a non-trivial object that mediates between the discretion of categorical diagrams (linked to transformational theory) and the continuity of gestures (linked to the performer's body). In the following section we discuss how abstract gestures are a conceptual unification between these discrete and continuous worlds of mathematical music theory.

\section{Abstract gestures AND the SIMPLicial APPROACH}

Though the immediate manifestation of gesturality that we perceive is linked to the human body and space, in the following lines we try to explain why the notion of gesturality is not spatial in essence. Mathematical details of this section are in [3, Chapter 3] and [7].

Diagrams in categories, which in a vague way can be considered as the diagrams that the mathematicians draw in the blackboard in courses of abstract algebra or homological algebra, are perhaps the most important non-spatial counterpart of bodily gestures. In turn, from a more musical point of view, diagrams of musical transformations, for example, affine transformations of musical modules, give rise to transformational theory, which studies the natural symmetries and correspondences that occur in music, and has important applications in musical analysis [26].

The notion of abstract gestures just establishes a dialogue between bodily gestures and diagrams in a natural and powerful way. Let us justify this strong assertion.

Both in topological gestures and diagrams in categories there is a common pattern. There is a skeleton or shape that represents an abstract configuration, which incarnates in a particular 


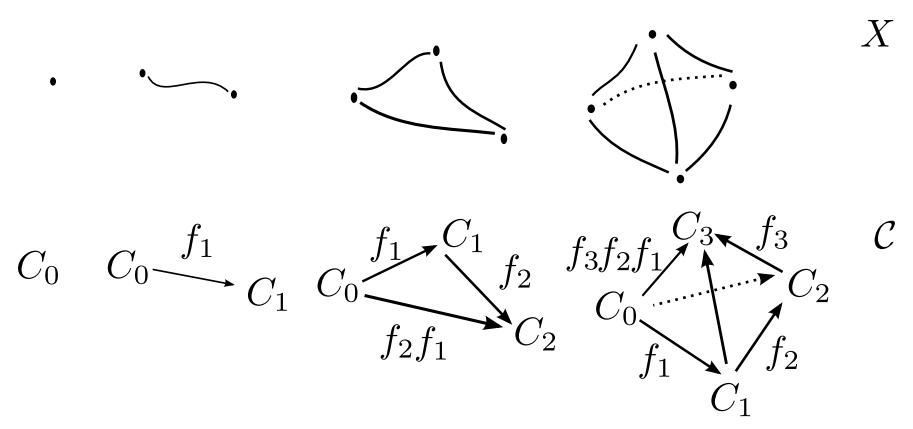

Figure 7: A point, a path, a triangle, and a tetrahedron in a space $X$, which are singular $n$-simplices for $n=0,1,2,3$ (first row). An object, a morphism, a commutative triangle, and a commutative tetrahedron in a category $\mathcal{C}$, which are $n$-simplices of the nerve for $n=0,1,2,3$ (second row).

context. In the case of topological gestures, the skeleta incarnate as paths and points in a space and, in the case of diagrams, skeleta become morphisms and objects in a category. Moreover, the existence of hypergestures and the need of a suitable model of the movement and configuration of the human body suggest that not only points and paths are important, but objects of higher dimensions are.

Poincaré's idea of studying a space by triangulation, leading to the concept of homology in a further stage of development (Section VII), is the base of our approach. Certainly, the singular complex of a space $X$ codifies all triangular objects of all dimensions that can occur in $X$ and their main relations (common faces, collapses). More formally, it is the presheaf $\operatorname{Top}\left(\Delta^{(-)}, X\right)$ on the simplicial category $\Delta$, where $\Delta^{(-)}$is the standard simplex functor, which assigns to each natural number $n$ all continuous maps $\Delta^{n} \longrightarrow X$ from the standard $n$-simplex to $X$, called singular $n$-simplices; see Figure 7. The singular complex is a combinatorial object whose abstraction leads to the idea of simplicial set, which is an abstract configuration of simplices (resembling singular ones) suitably pasted. Formally, a simplicial set is a presheaf on $\Delta$. With these formal tools, we define a $\Sigma$-gesture in $X$ as a natural transformation of simplicial sets from a simplicial set $\Sigma$, representing the abstract configuration of the gesture, to $\operatorname{Top}\left(\Delta^{(-)}, X\right)$, representing the possible incarnations in the space $X$. The natural transformation condition ensures that the relations that exist between abstract simplices in $\Sigma$ are preserved by their incarnations in $X$.

Following this definition, given a digraph $\Gamma$, it has a natural extension to a simplicial set $\Sigma$, whose associated notion of gesture is essentially that given by Mazzola since there is a natural bijection ${ }^{14}$ between the set of $\Gamma$-gestures (topological gestures) in $X$ and that of $\Sigma$-gestures in $X$, so the simplicial definition generalizes Mazzola's one. However, our definition immediately models the human body by taking a simplicial set $\Sigma$ that triangulates it. Figure 8 (left-hand side) reproduces a sculpture $\Sigma$ by Xavier Veilhan, which models the human body by means of abstract tetrahedra. A suitable $\Sigma$-shaped gesture in $\mathbb{R}^{3}$ is a human body, as shown in Figure 8.

From the categorical point of view, Grothendieck translated Poincaré's idea into the concept of nerve of a category. It codifies all possible strings of composable morphisms, their compositions (faces), and their extensions by using identities (degeneracies). More formally, the nerve of a category $\mathcal{C}$ is the presheaf $N(\mathcal{C})$ on $\Delta$ that assigns to each natural number $n$ all strings of composable morphisms in $\mathcal{C}$ of the form described in Equation 2:

$$
C_{0} \stackrel{f_{1}}{\rightarrow} C_{1} \stackrel{f_{2}}{\rightarrow} \ldots C_{n-1} \stackrel{f_{n}}{\rightarrow} C_{n}
$$

\footnotetext{
${ }^{14}$ Moreover, there is a natural homeomorphism [3, Proposition 3.5.2] between the space of gestures (digraph case) Г@X and the space of gestures for simplicial sets $S \pitchfork \operatorname{Top}\left(\Delta^{(-)}, X\right)$ defined below.
} 

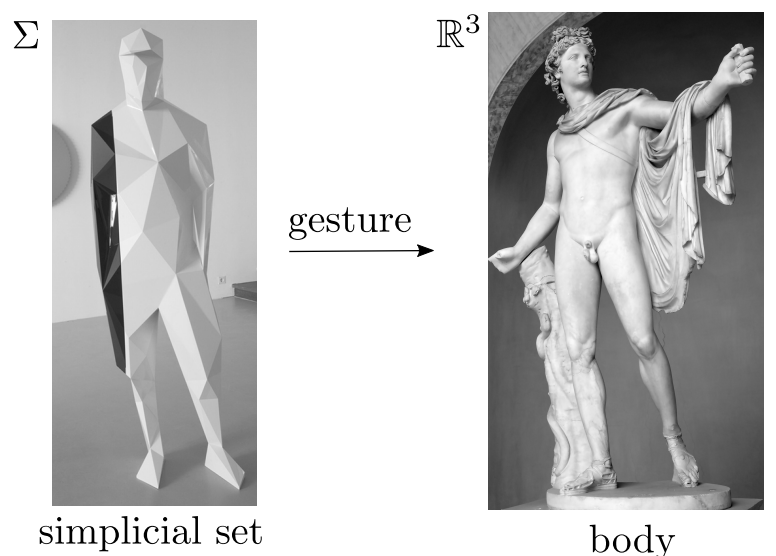

Figure 8: A human body, represented by means of the Apollo Belvedere (right-hand side), can be regarded as a gesture (natural transformation) shaped by a Veilhan's sculpture (left-hand side).

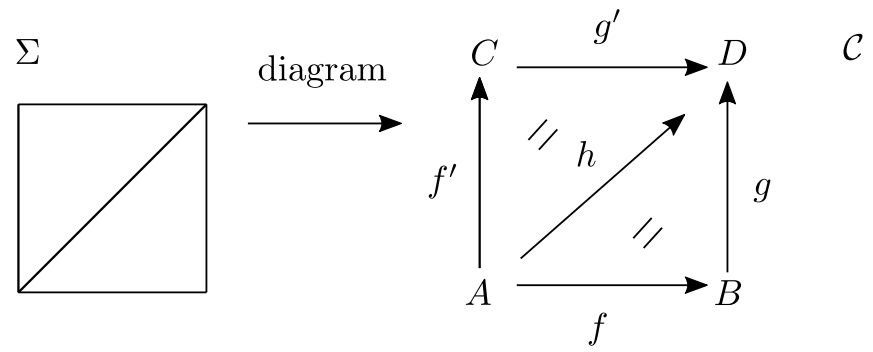

Figure 9: A commutative square as a diagram shaped by a suitable simplicial set.

These strings, which are the $n$-simplices, can be regarded as abstract triangulations, as displayed in Figure 7. In this case, the analogue of a gesture is a natural transformation from a simplicial set $\Sigma$ to the nerve, which sends vertices (0-simplices) and arrows (1-simplices) of $\Sigma$ to objects and morphism of $\mathcal{C}$, the relations expressed by simplices of higher dimensions being translated into different levels of commutativity relations. This analogue is just a (possibly) commutative diagram in $\mathcal{C}$; see Figure 9. In particular, when $\mathcal{C}$ is the category of modules on a commutative ring with affine morphisms between them, which include the natural symmetries that occur in music like transposition and inversion, we obtain the diagrams of transformational theory [26].

We thus conclude that the introduction of simplicial sets in the definition of gesture solves in a simple and unified way, on the one hand, the problem of the higher-dimensional representation of the human body in the topological world and, in the categorical world, the problem of considering diagrams with possible commutativity relations for transformational theory.

We have discussed gestures in the categories of topological spaces, topological categories, locales, categories, and Grothendieck topoi. This justifies a more comprehensive definition of gestures. The notion that unifies all the previous manifestations is that of abstract gestures. We define an object of gestures, shaped by a simplicial set, in a given category. In mathematical terms, given a simplicial set $\Sigma$ and a simplicial object $S$ (presheaf on $\Delta$ with values in a category $\mathcal{E}$ ), the object of $\Sigma$-gestures with respect to $S$ is the cotensor product $\Sigma \pitchfork S$, which is an object of $\mathcal{E}$, whenever it exists. The most important features of this object are the following:

- As a cotensor product, it has an associated adjunction and a universal property. This means 
that it is a categorical concept that has an intrinsic naturality.

- It is the dual concept of that of tensor product, and more precisely, for the presence of the simplicial category, it is the dual of the realization concept. In the case of topological spaces, the latter is the geometric realization of a simplicial set $\Sigma$, which transforms the combinatorial model $\Sigma$ of a space into the space. Thus, the concept of gestures is the dual of realization.

- We do not define a gesture as a primitive notion, since the objects of the category $\mathcal{E}$ need not have points, like locales.

- However, this definition includes the previous ones of individual topological gestures and diagrams. Topological gestures are elements (points) of the space of gestures $\Sigma \pitchfork$ $\operatorname{Top}\left(\Delta^{(-)}, X\right)$, where the singular complex is regarded as a presheaf whose values are topological spaces (function spaces). Diagrams are objects (points) of the category of gestures $\Sigma \pitchfork N(\mathcal{C})$, where the nerve is regarded as a presheaf whose values are categories functor categories).

Therefore, gesturality is not inherent to spaces, but can be found in any category.

\section{SHEAVES OF GESTURES}

The profound notion of sheaf embraces the notions of number and magnitude. At the same time, it unifies the visions of Galois and Riemann, as follows. On the one hand, the notion of scheme, a suitable gluing of affine schemes (sheaves on the prime spectrum of a commutative and unitary ring whose fibers are local rings), can be regarded as a generalization of that of algebraic variety (number) in the line of Galois-Hilbert-Zariski. On the other hand, the notion of Grothendieck topos, which is made up of sheaves that resemble the sheaf of germs of holomorphic functions in complex analysis, can be regarded as a generalization of that of topological space (magnitude) in the line of Riemann-Leray-Cartan. A great discovery of Grothendieck is that the notion of sheaf is not inherent to topological spaces but it can be formulated in terms of coverings of morphisms in any category, leading to Grothendieck topologies.

In Fernando Zalamea's words, sheaves are the simplest mathematical structures that allow the transit from the local to the global. Given a site, a sheaf is (informally) a correspondence assigning to each object $C$ of the category a set, whose elements are called sections over $C$, and to each morphism a restriction function, in such a way that coherent families (under restriction) of sections on the domains of morphisms of a covering of the Grothendieck topology glue together to form a unique section. The main application of sheaves (in Grothendieck's sense) to our discussion is that gestures can be glued together to produce new ones, thus forming sheaves [3, Theorem 3.12.1]. In mathematical terms, this means that the presheaf of gestures $(-) \pitchfork S:(\widehat{\Delta})^{o p} \longrightarrow \mathcal{E}$ obtained from the object of gestures construction, where $\widehat{\Delta}$ is the category of simplicial sets, is a sheaf for the Grothendieck topology of epimorphic sieves.

We can interpret the sheaf of topological gestures as follows. If we have a cover of a skeleton by skeleta that project exactly on it and a coherent family (indexed by the skeleta of the cover) of gestures, then the family yields a unique gesture, built from the initial family. This is an endless process that extends throughout all possible skeleta. We represent a fragment of the sheaf of gestures by means of Figure 10 .

Example 4. The construction of a global gesture from local ones can be observed in the fourth variation in Mozart's K. 331; see Figure 11. The melody is obtained by taking the harmonic tones in the theme (Example 1 and Figure 4) and a melodic and rhythmic uniformization. Remarkably, 

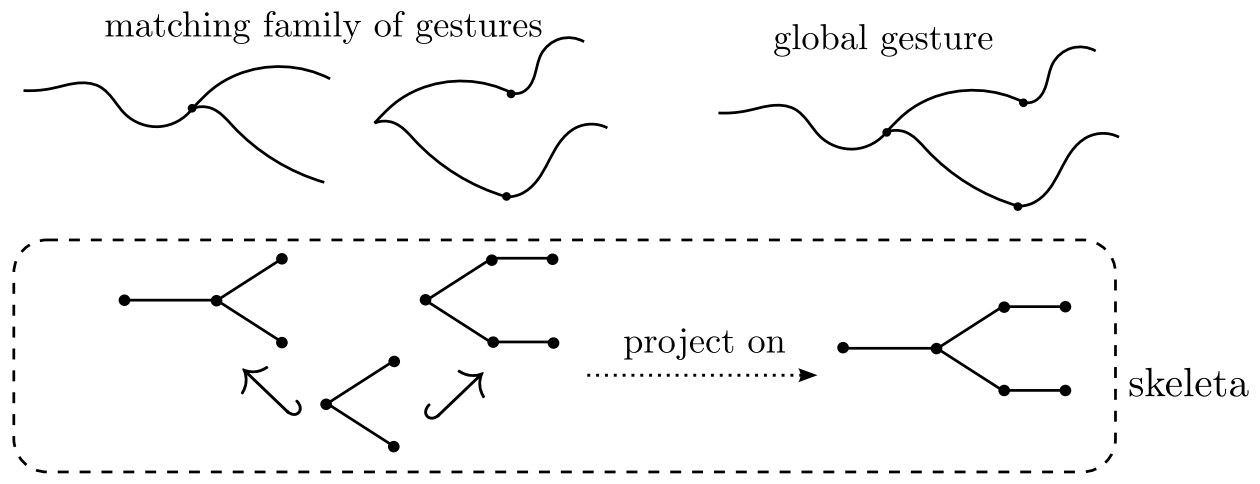

Figure 10: The sheaf of gestures: local gestures that are compatible extend to global ones.

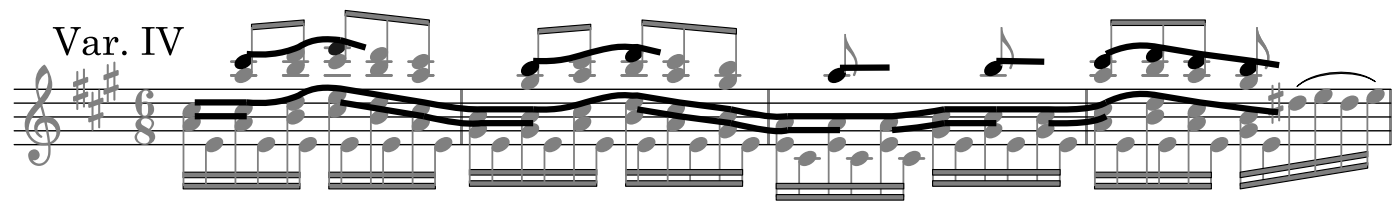

Figure 11: A global gesture in the fourth variation in Mozart's K. 331 (middle) from local melodic gestures (top and bottom). The harmonic tones are the black dots. We omit the digraphs, which are of the form $\bullet \rightarrow \bullet \cdots \bullet \rightarrow \bullet$, for simplicity.

the melodic fragments (gestures) in the top of each measure are coherently pasted (transposed by an octave) to form a global gesture that accompanies the melody.

Conversely, the definition of sheaves can be given in terms of cotensor products [3, Section 3.12]. Probably, this could express that $a$ sheaf is made up from gestures: in fact, for each object of the category, each covering of it, and each coherent family, a sheaf expresses a sort of movement of individual sections (limbs), with the configuration given by the covering involved, an objective (to form a section), and a modality (uniqueness of the section). In this way, sheaves can be related to the human gestures of solidarity and will, which oppose chaos.

The existence of sheaves of gestures also has a philosophical interpretation because it gives a precise mathematical proof of a previous intuition due to Cavaillès: the importance and possibility of multiplication of gestures, their solidarity, and their analytic continuation in Riemann's sense, as basis of understanding. Recalling the recurrent reference to Cavaillès in Mazzola's works [27]: "Comprendre est attraper le geste et pouvoir continuer."

Presheaves of gestures or gestural presheaves [29, Section 62.7] were also used to give a version of Yoneda lemma [29, Theorem 39] for gestures on topological categories. However, the result gives an embedding of categorical digraphs (analogue to topological and localic digraphs) of topological categories into gestural presheaves, which need not guarantee an embedding of topological categories into gestural presheaves.

Finally, there is another interesting way of addressing the problem of the local/global in gesture theory, namely the global gesture construction [29, Section 66.5] for gestures on topological categories, which is analogue to the global composition concept [29, Section 66.1]. In this case we give a cover of a digraph and a different gesture shaped by each member of the cover. But in this case the gestures are not defined in the same topological category, so we paste them by means of isomorphisms between the topological categories involved. 

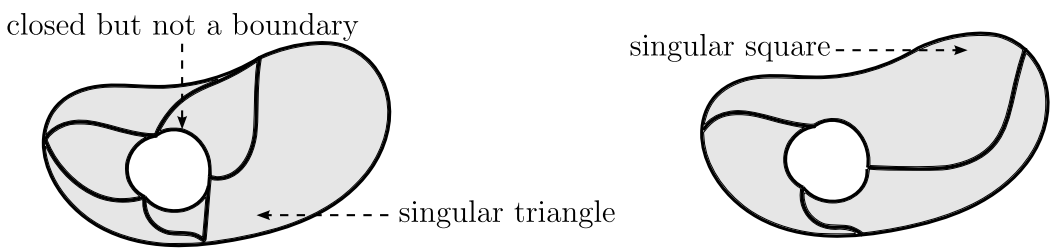

Figure 12: Triangulation (left-hand side) and squaring (right-hand side) of a surface with a hole.

\section{Homology}

Singular homology of a topological space is a tool created by Poincaré [34] so as to associate with the space certain invariants under continuous deformation (homotopy), namely Betti numbers, which are, intuitively, numbers of holes of different dimensions in the space. The development of this concept gave rise to the powerful language of homological algebra, which allows the correct formalization of Poincaré's ideas (homology modules) and the effective computation of Betti numbers. See [12] for a detailed account of the birth of homology theory. Later, the study of the dual notion of homology, that is, cohomology, led Eilenberg and Mac Lane to discover category theory via natural transformations; see the notes in [17, p. 29]. In fact, category theory is the natural environment of homological algebra, as witnessed, for example, by Grothendieck's Tôhoku paper [15], which achieved a unified treatment of (co)homology.

If the space is a surface in Euclidean space, the idea of homology is to perform a triangulation of the surface, that is, an approximation by means of simpler objects, by using singular triangles (in the surface) so as to detect holes by identifying closed trajectories that are not boundaries of singular triangles; see the left-hand side of Figure 12. Thus, homology can be regarded as a measure of how far a space is from being an ideal object (triangle). The procedure is generalized to solids, by detecting holes with tetrahedra, and then to higher dimensions, by using simplices.

However, these ideal objects can be changed, so as to produce other homologies. For example, if we consider squares (Figure 12, right-hand side) and cubes instead of triangles and tetrahedra, we will arrive to a homology that is essentially equivalent to the first. After all, these objects are equivalent for the purpose of detecting holes.

Another way of interpreting homology is inspired by the Yoneda lemma: we take a good subset of (ideal) domains to try to classify objects. For the category of sets, it is sufficient to consider the singleton domain, for digraphs it is the singletons and the one-arrow object. In homology, we try to classify spaces by using domains that are simplices.

Now, squares and cubes are instances of hypergestures in dimensions 2 and 3, so we could use general hypergestures to define homology [25]; see Figure 13. We would hope to gain new invariants of a space that were not grasped by classical homology or different computations of the latter by gestural means.

But hypergestural homology has a structural drawback. It is a particular case of cubical homology of a certain semi-cubical module, so it should be refined by means of a certain normalization process, based on degeneracies, so as to obtain trivial homology modules of contractible spaces. This point remains obscure since we have not a clear definition of degenerate hypergestures in this context. Some alternative definitions of hypergestural homologies, which are perhaps more natural, were given in [8] but they share the same problem.

The fact that simplicial homology does not require a normalization process, and that we have defined higher-dimensional gestures in the simplicial context, are powerful enough reasons to try a notion of simplicial gestural homology. This notion, as well as that of infinity-category of 

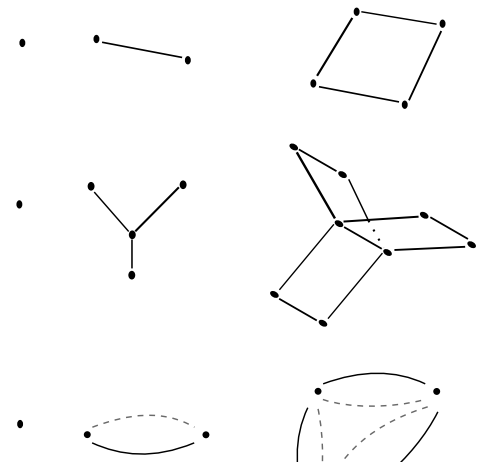
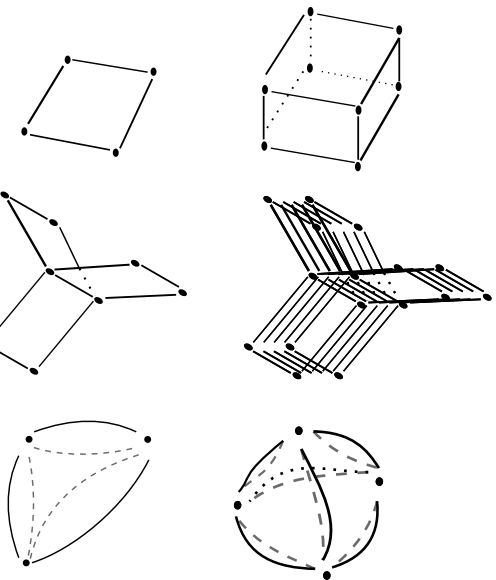

Figure 13: Hypercubes (first row). Hypergestures (second row). Gestures of higher dimensions, in the simplicial approach, associated with a certain cosimplicial space (third row). Compare with Figure 7.

gestures, is based on the observation that singular simplices can be regarded as gestures, under our definition in Section V. In fact, each continuous map $\Delta^{n} \longrightarrow X$ corresponds to a unique $\mathbf{y}_{n}$-gesture in $X$, where $\mathbf{y}_{n}$ is the combinatorial simplicial set modeling the space $\Delta^{n}$. Thus, classical simplicial homology is gestural and can be generalized by changing the models $\mathbf{y}_{n}$ for more general shapes. This leads to the notion of cosimplicial space (a functor $T: \Delta \longrightarrow \widehat{\Delta}$ ), which generalizes the Yoneda embedding $\mathbf{y}: \Delta \longrightarrow \widehat{\Delta}$ whose values are all models $\mathbf{y}_{n}$ for $n \in \mathbb{N}$, and hence to a notion of gestural homology, defined as the homology of the simplicial set $\widehat{\Delta}\left(T(-), \operatorname{Top}\left(\Delta^{(-)}, X\right)\right)$ called gestural complex, for each cosimplicial space $T$. Some gestures of higher dimensions, which generalize the singular simplices from Figure 7, are pictured in Figure 13.

At once this notion of homology is invariant under homotopy equivalence of spaces, and hence the homology modules of contractible spaces are trivial since gestural homology modules of the point space are. Details in [7].

\section{VIII. Номотору}

There are two main facts that lead to the study of simplicial homotopy applied to gesture theory. First, thanks to the generalization of gestures to the simplicial context, we can capture more retractions between (digraph-shaped) spaces and locales of gestures. This is due to the fact that by extending a digraph to a simplicial set we have identity arrows and they help collapse many digraphs that were not contractible with plain morphisms of digraphs. An important application of this fact is the determination of new examples of non-spatial locales of gestures, by finding retractions of them to familiar non-spatial locales. ${ }^{15}$ Second, the invariance of gestural homology with respect to homotopy equivalence of spaces, as a particular case of simplicial homology, can be obtained via simplicial homotopies of gestural complexes induced by homotopy equivalences of spaces. ${ }^{16}$ See [7] for details.

Example 5. Let us apply the simplicial retraction to the first movement in Mozart's K. 331.

\footnotetext{
${ }^{15}$ This reasoning is based on the fact that spatial locales are closed under retracts, so if the retract of a locale is non-spatial, then the original locale is non-spatial.

${ }^{16}$ More precisely, a homotopy equivalence of spaces induces a homotopy equivalence of simplicial sets (gestural complexes), which, in turn, induces a homotopy of chain complexes and isomorphisms of homology modules.
} 


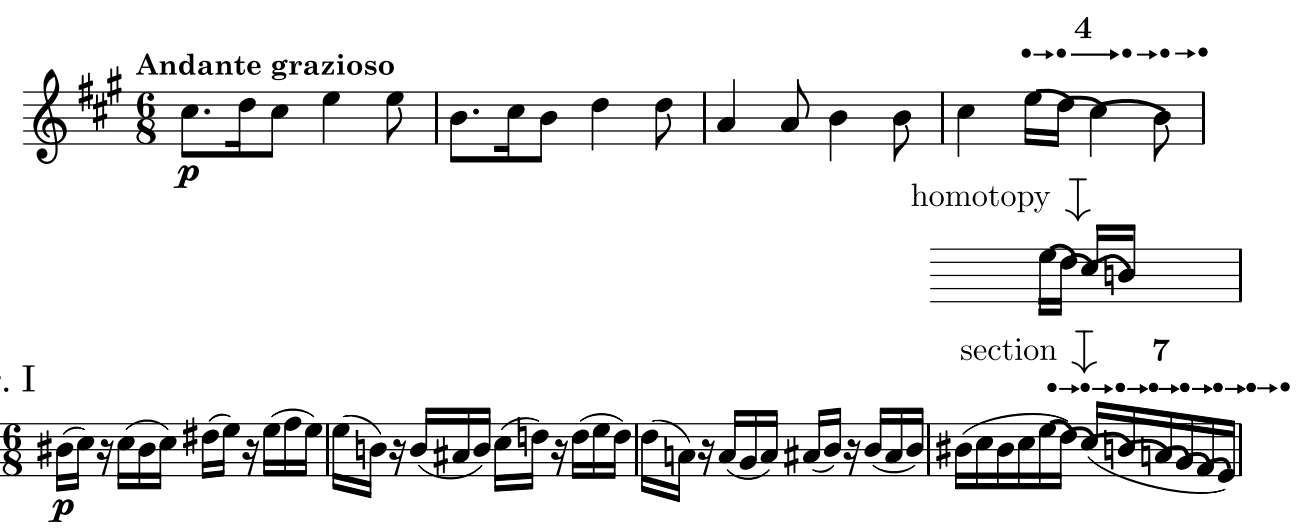

Figure 14: The descending gesture of the first variation in Mozart's K. 331 as an extension, by homotopy and section, of the original one.

First, consider the vertex digraph $\bullet$ and the arrow digraph $\bullet \rightarrow \bullet$. The extension (Definition in [7, Section 2.3]) to simplicial sets of these digraphs are the representable preshehaves $\Delta(-,[0])$ and $\Delta(-,[1])$ on the simplical category $\Delta$, the former being the final object in the category of simplicial sets. Moreover, we have that the topological spaces of gestures $\Gamma @ X$ and $L(\Gamma) \pitchfork S_{X}$ (where $L(\Gamma)$ is the simplicial extension of $\Gamma$ ) are homeomorphic [3, Theorem 3.5.2] for each digraph $\Gamma$ and each topological space $X$, and in our cases they are the space of continuous paths $X^{I}$ and $X$, respectively. The unique natural transformation $!: \Delta(-,[1]) \longrightarrow \Delta(-,[0])$ induces a continuous map (the presheaf functor $(-) \pitchfork S_{X}$ is a contravariant one to topological spaces) $f: X=\Delta(-,[0]) \pitchfork S_{X} \longrightarrow X^{I}=\Delta(-,[1]) \pitchfork S_{X}$. However, the same reasoning is not possible with digraphs since there is no digraph morphism from $\bullet \rightarrow \bullet$ to $\bullet$; it must send arrows to arrows, but the bullet digraph has no arrows.

Actually, the map $f$ sends an element $x \in X$ to its constant path with value $x$ and has as left inverse the evaluation at 0 map. This means that $f$ is a section. Similarly, we can find sections from $\mathbf{n} @ X$ to $\mathbf{m} @ X$, where $n<m$ in $\mathbb{N}$, and $\mathbf{n}$ denotes the digraph $\bullet \rightarrow \cdots \rightarrow \bullet$ with $n$ arrows.

In the first variation in Mozart's K. 331, fourth measure, we can observe how the original descending gesture in the score space (Example 1) is extended; see Figure 14. First, the last two notes ( $\mathrm{C} \sharp$ and $\mathrm{B})$ suffer a contraction in their durations, which is just a continuous path, or homotopy, in the space $4 @ \mathbb{R}^{2}$. Then the descending gesture in the last measure extends to one in $7 @ \mathbb{R}^{2}$ by means of sections and homotopies.

\section{i. Infinity-categories of gestures}

Infinity-categories are a convenient language for categories with morphisms of higher dimensions, avoiding the long lists of data and axioms that are usually needed for defining them. For instance, in the 3-category of 2-categories we have pseudofunctors (relating categories), pseudonatural transformations (relating pseudofunctors), modifications (relating pseudonatural transformations), and a lot of compatibility axioms between them. On the other hand, in a topological space, we have elements, paths linking elements, path homotopies, and so on, together with notions of composition. These two analogous manifestations can be synthesized in the definition of infinity-category, which is a simplicial set satisfying certain lifting properties that allow to define the usual categorical operations.

In particular, the two most important simplicial sets that we considered in our discussion of 
abstract gestures (Section V) are examples of infinity-categories: the nerve of a category, related to possibly commutative diagrams (gestures) for transformational theory, and the singular complex, related to the performer's gestures.

Example 6. This example is inspired by [20,19]. Let $\Sigma$ be a simplicial set modelling a human body (Figure 8). Consider a topological space $X$, where the movement of the body occurs (for example, a subspace of the usual Euclidean space $\mathbb{R}^{3}$ ), and the topological space $\Sigma \pitchfork S_{X}$ of all $\Gamma$-gestures in $X$ (Section V). The singular complex $\operatorname{Top}\left(\Delta^{(-)}, \Sigma \pitchfork S_{X}\right)$ is an example of $\infty$-category.

The categorical structure is the following. Objects are $\Sigma$-gestures in $X$, which are just poses of the body. The 1-morphisms are continuous paths of poses in $\Sigma \pitchfork S_{X}$, which can be interpreted as movements of the body. The 2-morphisms are paths in the path space $\left(\Sigma \pitchfork S_{X}\right)^{I}$, that is, paths between movements of the body, etc. The composition of morphisms is carried out modulo homotopy equivalence.

Based on cosimplicial spaces, we can construct many gestural complexes (Section VII). Since the singular complex is a particular case of a gestural complex, this suggests that, under suitable conditions, gestural complexes can be $\infty$-categories. In such cases, we have a notion of composition of gestures, which was an initial motivation for introducing $\infty$-categories in the gestural context [4, p. 103]. Several examples of $\infty$-groupoids ${ }^{17}$ of gestures, other than the singular complex, are given in [7].

The introduction of infinity-categories in gesture theory also responds to Mazzola's fundamental problem of rebuilding algebraic operations from gestural instances. In [27, Section 6.1], the fundamental groupoid of a space was acknowledged as an important tool for reconstructing a certain gestural substance (based on loops and their homotopies) behind group operations. In a more general way, $\infty$-groupoids, $\infty$-categories, and, in particular, categories could be identified as $\infty$-categories of gestures, thus providing fully gestural information on their algebraic operations. For example, by a central theorem of Quillen [14, Theorem 11.4], we know that in an appropriate sense, each $\infty$-groupoid is equivalent to the singular complex of a certain topological space, so we can regard operations in an $\infty$-groupoid in terms of homotopies related to gestures shaped by combinatorial simplices.

\section{APPLICATIONS AND RELATIONS TO OTHER FIELDS}

\section{i. Mathematics}

Within mathematics we have the following incidences of gesture theory.

\section{Locales}

In locale theory, a way of exhibiting examples of non-spatial locales is to take suitable exponentials and limits. Since the construction of locales of gestures requires these constructions, many locales of gestures are non-spatial. Thus, gestures are useful for providing examples of non-spatial locales.

\section{Realizations}

It is very curious that, unlike the realization concept, its dual (gestures) has no substantial reference in the categorical literature, up to the knowledge of the authors. For example, geometric

\footnotetext{
${ }^{17}$ Informally, $\infty$-groupoids are $\infty$-categories whose morphisms are invertible.
} 
realizations and fundamental categories are the realizations associated with the singular complex and nerve functors, but the respective gestural notions are overlooked.

Realizations are central in abstract homotopy theory. Besides the importance of geometric realizations, fundamental categories of simplicial sets are a basic tool for understanding the usual categorical structure behind $\infty$-categories.

\section{Infinity-categories}

Some gestural complexes (Section VII) are examples of infinity-groupoids that have a topological flavor, but that are not singular complexes, as shown in [7].

\section{Sheaves}

The definition of sheaf can be given in terms of cotensor products (see Section VI), which suggests a profound relation between sheaves and gestures.

\section{Homology}

By [31, Lemma 5], each gestural homology (Section VII) is equivalent to the classical homology of a certain space. This and the plasticity of gestures suggest that we could use gestural homology to compute classical homology modules. For example, in [7], there is a simplified proof of a Hurewicz theorem based on gestural homology.

\section{ii. Mathematical music theory}

In mathematical music theory, where gestures were conceived, we have the following connections.

\section{Transformational theory}

Diagrams are abstract gestures (Section V). Also, the topological structure of the topological category of continuous paths of a digraph [6] can help understand classical diagrams as continuous processes, near to Mazzola's ideal of recovering gestures from morphisms.

\section{Counterpoint theory}

One of the main notions of counterpoint, and of Mazzola's model, is that of interval. Mazzola's intuition of intervals as gestures is supported by the fact that there is an infinity-groupoid of intervals, which links intervals to paths, via Quillen's vision of infinity-groupoids as singular complexes. Moreover, this groupoid is useful for determining when a partition $\{K, D\}$ of the ring of intervals $\mathbb{Z}_{12}$ has an affine symmetry. This is important, since the existence of such a symmetry is necessary to open up a non-traditional counterpoint world based on consonances (in $K$ ) and dissonances (in $D$ ). A recent exposition of mathematical counterpoint theory is [5].

\section{OPEN AND CLOSED PROBLEMS}

\section{The diamond conjecture}

In essence, the diamond conjecture asserts the existence of an adjunction between the category of topological gestures and the category of formulas, the latter understood as diagrams of 
transformational theory. The adjunction is the composition of two adjunctions linked by a category that would offer a unified comprehension of music [27, Section 9].

However, there are several technical reasons that suggest a review of Mazzola's notion of formula [3, Section 3.11.2]. More generally, the notion of abstract gestures is already a concept that unifies all notions of spatial gestures, and transformational diagrams. Hence, the statement of the conjecture could be revisited under this notion. However, the conjecture surmises the existence of a unifying object, which has not been found up to now.

Gestural Yoneda lemma and the problem of rebuilding categories and operations from gestures

As commented before, a solution has been proposed in [29, Section 62.7], in the context of topological categories. The generalization of the representation theorem of categorical digraphs [29, Theorem 39] may be explored for abstract gestures. It is also an open problem whether the notion of gestures on Grothendieck topoi is useful to provide such a lemma. The existence of infinity-categories of gestures also suggests the possibility of rebuilding operations in (infinity)categories from gestural movements. This deserves a serious study.

\section{The problem of rebuilding topological gestures from algebraic data}

Locales help address the problem of representing the movement of the human body in algebraic terms. In turn, this can be helpful for introducing gestures in a computational language. In fact, the topology of every space of gestures on a sober space is embedded in a locale of gestures, as shown in [3, p. 18], and each individual gesture is a point of a certain locale of gestures [3, Corollary 2.4.3], locales being particular cases of lattices.

The characterization of the singular complex and more general gestural complexes as infinitycategories also shows that gestural movements can be managed at the level of categorical operations. Other approaches to this problem could be considered.

\section{Exponential presentation of objects of gestures}

This problem is inspired by the fact that each topological space of gestures, in the case of digraphs, can be regarded as a function space whose domain is the geometric realization of the digraph involved [4, Theorem 7.3]. Similarly, the category of $\Gamma$-shaped diagrams, for $\Gamma$ digraph, can be regarded as a category of functors whose common domain is the category of paths of $\Gamma$, the latter being a realization in the category of categories [3, p. 81].

The exponential presentation problem consists in determining whether the object of $\Sigma$-gestures in an object $C$ of a category is the exponential $C^{|\Sigma|}$ for a certain realization ${ }^{18}|\Sigma|$, where $\Sigma$ is a digraph, or a (semi-)simplicial set. It has been solved in the cases of semi-simplicial sets $\Sigma$ for topological spaces and the geometric realization, of digraphs $\Sigma$ for locales and the induced geometric realization, and for cartesian closed categories; see [3, Section 3.2]. The case of topological categories was initially studied in [6] without a full solution, but with a characterization of topological categories of gestures as topological categories of functors, whose domains are categories of continuous paths of digraphs. It is also worth asking whether this problem can be addressed in a general framework that does not depend on a particular category.

${ }^{18}$ The realization usually has better homotopical properties than the skeleton $\Sigma$. 


\section{Normalization of hypergestural homologies}

A notion of normalization for hypergestural homology would pave the way for a more complete study of it, including its computation and relation to classical homology. It is not clear whether hypergestural homology provides more information about an space beyond classical homology. See [8].

\section{Gestural homologies}

In the simplicial case, it is an open problem the explicit computation of gestural homologies, which are based on particular simplicial sets, namely gestural complexes. Also, it is an open problem whether gestural homologies offer simplified computations of classical homologies. Details in [7].

\section{Spatiality of locales of gestures}

In essence, all locales of gestures on a non-spatial locale are non-spatial [3, Corollary 2.5.3]. Thus, the problem of spatiality reduces to studying locales of gestures on spatial locales. There are several possibilities, depending on the space and the digraphs. A summary of results can be found in $[3$, Section 2.9]. The most important concepts used were exponential presentation, which is specially linked to the theory of injective locales, and retractions.

Some improvements on the preceding results were made by means of the extension of digraphs to simplicial sets and the introduction of simplicial retractions [7]. Nevertheless, a complete theory is far from being completed.

\section{Infinity-categories}

Up to now, we do not know if there are gestural complexes that are examples of infinity-categories but not infinity-groupoids [7].

\section{Philosophy}

The discussion in Section II together with [29, Part XV] can give rise to a lot of philosophical developments on gestures.

\section{ENVIRONMENT}

The place of gesture theory, and more generally mathematical music theory, is privileged in knowledge but usually misunderstood by pure mathematicians and musicians. Some mathematicians could regard mathematical music theory as a not serious one or as a branch that is not of general interest for the mathematical community. On the other hand, musicians could assess this theory as excessively formal or even useless.

However, it is undeniable that mathematics and music can benefit each other. There are many mathematical tools, such as group theory, that have a long tradition of use among composers. Also, as the cases of gestures and Grothendieck's motifs [21] show, there are musically motivated concepts that can bear a considerable mathematical interest. Mathematics is an infinite source of tools for composing and understanding music. Music is an infinite source of inspiration for doing mathematics. In this way, the study of the relationship between mathematics and music is important for human knowledge since it helps understand creativity and multiply it in both fields. 
And gestures precisely allow the dialogue between mathematics and music as human activities. Recalling Mazzola's fundamental dialectic [23, Chapter 1]: the performer's gestures, inspired by scores, become real music and the mathematician's gestural intuitions become theorems. Every human activity is a gesture. Everyday technology is full of gestural devices. Consequently, gesture theory should be the easiest branch of mathematical music theory to share with the public.

\section{GLOSSARY}

In what follows $\mathcal{C}, \mathcal{D}$, and $\mathcal{E}$ denote arbitrary categories.

Free object Let $U: \mathcal{C} \longrightarrow \mathcal{D}$ be a functor. Given an object $D$ of $\mathcal{D}$, we say that an object $F(D)$ of $\mathcal{C}$ is a free or universal object of $D$ if there is a morphism $\eta: D \longrightarrow U F(D)$ such that for each morphism $f: D \longrightarrow U(C)$ there is a unique $h: F(D) \longrightarrow C$ such that $U(h) \eta=f$. The latter condition is called universal property of $F(D)$. Examples: if $U$ is the forgetful functor from abelian groups (respectively topological spaces) to sets, $F(X)$ is the free abelian group on $X$ (respectively $X$ with the discrete topology).

Topological gesture A digraph $\Gamma$ is a quadruple $(A, V, t, h)$ such that $A$ and $V$ are sets whose elements are called arrows and vertices, respectively. Given a topological space $X$, we can construct the topological digraph $\vec{X}$ of $X$, which is the quadruple $\left(X^{I}, X, e_{0}, e_{1}\right)$, where $I$ is the usual interval $[0,1]$ in $\mathbb{R}, X^{I}$ is the space of continuous paths $c: I \longrightarrow X$ with the compact-open topology, and $e_{0}$ and $e_{1}$ are the continuous evaluation maps at 0 and 1 . A $\Gamma$-gesture in $X$ is a digraph morphism from $\Gamma$ to $\vec{X}$. It consists of two functions $u: A \longrightarrow X^{I}$ and $v: V \longrightarrow X$ such that $e_{0} u=v t$ and $e_{1} u=v h$.

Compact-open topology The subbasic opens of the compact-open topology on the space of continuous paths $X^{I}$ are those of the form $\{c: I \longrightarrow X$ continuous $\mid c(K) \subseteq U\}$, where $K$ is compact (closed) in $I$ and $U$ is open in $X$. This makes $X^{I}$ an exponential in the category of topological spaces.

Space of gestures Let $\Gamma$ and $X$ be as in the topological gesture definition. The topological space of all $\Gamma$-gestures in $X$, denoted by $\Gamma @ X$, is the limit in the category of topological spaces of the diagram consisting of for each $a \in A$ a copy of $X^{I}$, for each $z \in V$ a copy of $X$, a copy of $e_{0}$ whenever $z=t(a)$, and a copy of $e_{1}$ whenever $z=h(a)$. The elements of the space $\Gamma @ X$ are in bijective correspondence with the respective topological gestures.

Locale Complete lattice satisfying the distributivity of $\wedge$ (meet) with respect to the infinitary $\vee$ (join). A morphism of locales from $L$ to $M$ is a function from $M$ to $L$ that preserves finite meets and arbitrary joins. Examples: 1. Given a topological space $X$, the poset of its opens $\mathcal{O}(X)$ is a locale. This construction defines a functor $\mathcal{O}$ from topological spaces to locales sending a continuous map $f: X \longrightarrow Y$ to the inverse image map $f^{-1}(-): \mathcal{O}(Y) \longrightarrow \mathcal{O}(X)$. 2. (Fundamental example) The lattice of subsheaves of any sheaf on a (small) site is a locale. In the case of the final sheaf (constant sheaf with value $\{*\}$ ), the subsheaves are the opens of the site, and hence the opens of any site form a locale.

Locale of gestures Let $\Gamma$ be a digraph with $\Gamma=(A, V, t, h)$ and $L$ a locale. Consider the continuous endpoint inclusions $i_{0}, i_{1}:\{*\} \longrightarrow I$ of the interval $I$ and the induced morphisms of locales $\mathcal{O}\left(i_{0}\right), \mathcal{O}\left(i_{1}\right): \mathbf{2} \longrightarrow \mathcal{O}(I)$, where 2 denotes the final locale. We can construct the localic digraph $\vec{L}$ 
of $L$, which is the quadruple $\left(L^{\mathcal{O}(I)}, L, e_{0}, e_{1}\right)$, where $L^{\mathcal{O}(I)}$ is an exponential ${ }^{19}$ locale, $e_{0}=L^{\mathcal{O}\left(i_{0}\right)}$, and $e_{1}=L^{\mathcal{O}\left(i_{1}\right)}$. The locale of all $\Gamma$-gestures in $L$, denoted by $\Gamma @ L$, is the limit in the category of locales of the diagram consisting of for each $a \in A$ a copy of $L^{\mathcal{O}(I)}$, for each $z \in V$ a copy of $L$, a copy of $e_{0}$ whenever $z=t(a)$, and a copy of $e_{1}$ whenever $z=h(a)$.

Point If $\mathcal{C}$ has a final object 1 , we define a point of an object $C$ of $\mathcal{C}$ as a morphism $\mathbf{1} \longrightarrow C$. Examples: 1. Points in the categories of sets and topological spaces correspond to elements. 2. Points of the locale $\mathcal{O}(X)$, where $X$ is a Hausdorff (or more generally, sober) space, are just elements of $X$.

Presheaf $A$ presheaf on a category $\mathcal{C}$ is a contravariant functor from $\mathcal{C}$ to a suitable ${ }^{20}$ category of sets. All presheaves on $\mathcal{C}$ form a category $\widehat{C}$ whose morphisms are natural transformations between presheaves. Examples: 1 . The representable functors, which are of the form $\mathcal{C}(-, C)$ for $C$ object of $\mathcal{C}$, are presheaves on $\mathcal{C}$. 2. Any simplicial set, which is a presheaf on the simplicial category. 3. The singular complex functor $\operatorname{Top}\left(\Delta^{(-)}, X\right)$ of a topological space. 4 . The nerve of a category $\mathcal{C}$ of a category $\mathcal{C}$, which is the presheaf $\operatorname{Cat}(-, \mathcal{C})$ on the simplicial category that sends $[n]$ to the functor category $\operatorname{Cat}([n], \mathcal{C})$ regarding $[n]$ as a poset category, and $\alpha:[n] \longrightarrow[m]$ to $(-) \circ \alpha: \operatorname{Cat}([m], \mathcal{C}) \longrightarrow \operatorname{Cat}([n], \mathcal{C})$ regarding $\alpha$ as a functor.

Yoneda lemma Given a presheaf $P$ on $\mathcal{C}$ and an object of $\mathcal{C}$, it establishes a natural bijection between the sets of natural transformations from $\mathcal{C}(-, C)$ to $P$ and $P(C)$. Explicitly, the bijection sends such a natural transformation $\tau$ to $\tau_{C}\left(i d_{C}\right)$.

Yoneda embedding The functor $\mathbf{y}: \mathcal{C} \longrightarrow \widehat{C}$ sending an object $C$ to the representable functor $\mathcal{C}(-, C)$ and a morphism $f: C \longrightarrow D$ to the natural transformation defined by composition with $f$ in each component. This functor is full and faithful by the Yoneda lemma.

Sieve Let $C$ be an object of a category $\mathcal{C}$. A sieve on $C$ is a set of morphisms with codomain $C$ that is closed under right composition. Examples: 1 . The maximal sieve $t(C)$ consisting of all morphisms with codomain $C$. 2 . The sieve generated by a set $X$ of morphisms with codomain $C$, defined as the closure of $X$ under right composition. 3. The restriction sieve $h^{*}(S)$ of a sieve $S$ on $C$ along a morphism $h: D \longrightarrow C$, defined as the set of all morphisms $f$ with codomain $D$ such that $h f$ is in $S$.

Grothendieck topology A Grothendieck topology J on a category $\mathcal{C}$ consists of for each object $C$ a set of covering sieves $J(C)$ such that i) the maximal sieve $t(C)$ is in $J(C)$, ii) if $S$ is in $J(C)$, then all possible restriction sieves of $S$ are covering sieves, and iii) if all possible restriction sieves of a given one $S$ are covering sieves, then $S$ is a covering sieve. Examples: 1. Let $T$ be a topology (of a topological space) regarded as a category (category of a poset). The sieves generated by open coverings of opens in $T$ are the covering sieves of a Grothendieck topology on T. 2. Consider the category $\widehat{C}$ of presheaves on $\mathcal{C}$. The epimorphic sieves of a presheaf $P$ are the sieves $S$ on $P$ such that for each object $C$ of $\mathcal{C}$ the set of images $\left\{\operatorname{Im}\left(\tau_{C}\right) \mid \tau \in S\right\}$ covers $P(C)$.

Site Category with a Grothendieck topology $(\mathcal{C}, J)$.

\footnotetext{
${ }^{19}$ The locale $\mathcal{O}(I)$ is exponentiable since it is a continuous lattice and hence this exponential and its derivative constructions exist. Also, the final object 2 is exponentiable (in any category) and the exponential $L^{2}$ is isomorphic to $L$. Details in [2] and [3, Section 2.3].

${ }^{20}$ That is, the sets are the members of a Grothendieck universe [9, Exposé I].
} 
Sheaf A presheaf $F$ on a site $(\mathcal{C}, J)$ is a sheaf if for each object $C$ of $\mathcal{C}$ and each covering sieve $S$ in $J(C)$, given a family of local sections $\left\{x_{f} \mid f\right.$ in $S$ and $\left.x_{f} \in F(\operatorname{dom}(f))\right\}$ such that $F(h)\left(x_{f}\right)=x_{f h}$ whenever the composite $f h$ exists, there is a unique $x \in F(C)$ such that $F(f)(x)=x_{f}$ for each $f$ in $S$. In such a case we say that $x$ is a global section. Examples: given the site of the usual topology of $\mathbb{R}$ (respectively $\mathbb{C}$ ), the presheaf with $P(U)$ defined as the set of all (continuous or differentiable) functions defined on $U$ with values in $\mathbb{R}$ (respectively $\mathbb{C}$ ) is a sheaf.

Grothendieck topos Category (equivalent to one) of sheaves on a site, which is a full subcategory of the associated category of presheaves. Examples: 1. All categories of presheaves are Grothendieck topoi since presheaves are sheaves on the trivial topology whose covering sieves are the maximal ones, in particular the categories of digraphs and semi-simplicial sets are. 2. Categories of sheaves on the examples of Grothendieck topologies.

Exponential presentation We can state the problem, in the case of simplicial sets (for short), as follows. Let $T: \Delta \longrightarrow \mathcal{C}$ be a functor, where $T$ has all its images exponentiable in $\mathcal{C}$. Given an object $C$ of $\mathcal{C}$, we define $S_{C}$ as the functor $C^{T(-)}: \Delta^{o p} \longrightarrow \mathcal{C}$. This object generalizes the internal versions of the singular complex and the nerve. Given a simplicial set $\Sigma$, we ask whether the object of gestures $\Sigma \pitchfork S_{C}$ coincides with the exponential $C^{|\Sigma|_{T}}$, where $|\Sigma|_{T}$ is the realization of $\Sigma$ with respect to $T$, whenever these objects exist. In the case when $\mathcal{C}$ is Cartesian closed, we solve the problem immediately, since $C^{(-)}$transforms the colimit $|\Sigma|_{T}$ into the limit $\Sigma \pitchfork S_{C}$. Otherwise, the solution is not guaranteed. See [3, Section 3.2] for details.

Simplicial category Denote by $[n]$ the ordered set (ordinal) $\{0,1, \ldots, n\}$ for $n \in \mathbb{N}$. The simplicial category $\Delta$ has as objects all $[n]$ for $n \in \mathbb{N}$ and as morphisms all order-preserving maps between them.

Standard simplex (functor) For each $n \in \mathbb{N}$, we define the standard $n$-simplex $\Delta^{n}$ as the set in Equation 3:

$$
\left\{\left(t_{1}, \ldots, t_{n}\right) \mid 0 \leq t_{1} \leq \cdots \leq t_{n} \leq 1\right\}
$$

The standard $n$-simplex is a subspace of $\mathbb{R}^{n}$ and this construction defines a standard simplex functor $\Delta^{(-)}$from the simplicial category to the category of topological spaces, which sends an order preserving map $\alpha:[n] \longrightarrow[m]$ to the appropriate continuous map $\Delta^{\alpha}: \Delta^{n} \longrightarrow \Delta^{m}$ sending the $i$ th vertex (with $n-i$ zeros) to the $\alpha(i)$ th one. Examples: $\Delta^{0}$ is a singleton, $\Delta^{1}$ is the interval $[0,1]$ in $\mathbb{R} ; \Delta^{2}$ is the triangle with vertices $(0,0),(0,1)$, and $(1,1)$ in $\mathbb{R}^{2}$; and $\Delta^{3}$ is the tetrahedron with vertices $(0,0,0),(0,0,1),(0,1,1)$, and $(1,1,1)$ in $\mathbb{R}^{3}$.

Tensor product Suppose that $\mathcal{E}$ has small hom-sets and small colimits and that $\mathcal{C}$ is small. Suppose given a functor $T: \mathcal{C} \longrightarrow \mathcal{E}$. Consider the functor $\mathcal{E}(T,-): \mathcal{E} \longrightarrow \widehat{\mathcal{C}}$ that sends $E$ to the presheaf $\mathcal{E}(T(-), E)$. The tensor product $P \otimes_{\mathcal{C}} T$, where $P$ is a presheaf on $\mathcal{C}$, is the free object of $P$ with respect to the functor $\mathcal{E}(T,-)$ if it exists. It can be computed via the colimit ${ }^{21}$ in Equation 4:

$$
\operatorname{Colim}\left(\int P \stackrel{\pi}{\rightarrow} \mathcal{C} \stackrel{T}{\rightarrow} \mathcal{E}\right)
$$

\footnotetext{
${ }^{21}$ Here, $\int P$ denotes the category of elements of $P$. Its objects are of the form $(C, p)$ where $p \in P(C)$ and $C$ is an object of $\mathcal{C}$. A morphism from $(C, p)$ to $\left(C^{\prime}, p^{\prime}\right)$ is a morphism $f$ of $\mathcal{C}$, from $C$ to $C^{\prime}$, such that $P(f)\left(p^{\prime}\right)=p$. We denote by $\pi: \int P \longrightarrow \mathcal{C}$ the natural projection functor.
} 
Actually, this construction defines a left adjoint $(-) \otimes_{\mathcal{C}} T$ to $\mathcal{E}(T(-), E)$, which means that there is a bijection, as in Equation 5:

$$
\mathcal{E}\left(P \otimes_{\mathcal{C}} T, E\right) \cong \widehat{\mathcal{C}}(P, \mathcal{E}(T(-), E)),
$$

natural in $P$ and E. Examples: 1 . If $\mathcal{C}$ is the simplicial category $\Delta$, the tensor product $\Sigma \otimes \Delta^{(-)}$, where $\Sigma$ is a simplicial set, is the realization $|\Sigma|_{T}$ of $\Sigma$ with respect to $T$. 2 . In the case when $T$ is the standard simplex functor $\Delta^{(-)}, \Sigma \otimes \Delta^{(-)}$is the geometric realization $|\Sigma|$ of $\Sigma$.

Cotensor product It is the dual of the tensor product notion-we change $\mathcal{E}$ for its opposite in the above definition. Thus, the difference is that now we require that $\mathcal{E}$ has small limits. We give a functor $S: \mathcal{C}^{o p} \longrightarrow \mathcal{E}$ and consider $\mathcal{E}(-, S): \mathcal{E} \longrightarrow(\widehat{\mathcal{C}})^{o p}$. The cotensor product $P \pitchfork S$, where $P$ is a presheaf on $\mathcal{C}$, is the limit in Equation 6:

$$
\operatorname{Lim}\left(\left(\int P\right)^{o p} \stackrel{\pi^{o p}}{\rightarrow} \mathcal{C}^{o p} \stackrel{S}{\rightarrow} \mathcal{E}\right)
$$

The corresponding natural bijection is given in Equation 7:

$$
\mathcal{E}(E, P \pitchfork S) \cong \widehat{\mathcal{C}}(P, \mathcal{E}(E, S)) .
$$

Example: If $\mathcal{C}$ is the simplicial category and $\Sigma$ is a simplicial set, then $\Sigma \pitchfork S$ is the object of $\Sigma$-gestures with respect to $S$. This notion includes all gesture definitions mentioned in this paper.

Simplicial homology Let $S$ be a simplicial set and $R$ a commutative ring. For each $n \in \mathbb{N}$, the $n$th homology of $S$ is the quotient $R$-module $\operatorname{Ker}\left(\partial_{n}\right) / \operatorname{Im}\left(\partial_{n+1}\right)$, where $\partial_{n}=\sum_{i=0}^{n}(-1)^{i} d_{i}$ for $n \geq 1$ and $d_{i}: R^{S[n]} \longrightarrow R^{S[n-1]}$ is the linear extension (to free modules) of the corresponding face of $S$. The $R$-homomorphism $\partial_{0}$ is the unique from $R^{S[0]}$ to the trivial module $\{0\}$. Example: The simplicial homology of the singular complex of a topological space $X$ is the classical homology of $X$.

Spatial locale A locale is spatial if it is isomorphic to that of opens $\mathcal{O}(X)$ of some topological space $X$. Otherwise we say that it is non-spatial.

Infinity-category Simplicial set $S$ such that given $n \in \mathbb{N}$ and $k$ with $0<k<n$, for each subset $\left\{a_{i} \mid 0 \leq i \leq n ; i \neq k\right\}$ of $S([n-1])$ satisfying the identities of Equation 8:

$$
d_{i}\left(a_{j}\right)=d_{j-1}\left(a_{i}\right) \quad(i<j ; i, j \neq k),
$$

there is an element $a \in S([n])$ such that $d_{i}(a)=a_{i}$ for $i \neq k$. If this property also holds for $k=0$ and $k=n$, then we say that $S$ is an $\infty$-groupoid. Examples: 1 . The nerve of a category is an $\infty$-category. 2 . The singular complex of a topological space is an $\infty$-groupoid.

\section{REFERENCES}

[1] Arias, J. 2017. Abstract Gestures: A Unifying Concept in Mathematical Music Theory. In: Mathematics and Computation in Music. Cham: Springer, pp. 183-200.

[2] Arias, J. 2017. Gestures on Locales and Localic Topoi. In: The Musical-Mathematical Mind: Patterns and Transformations. Cham: Springer, pp. 29-39. 
[3] Arias, J. 2018. Gesture Theory: Topos-Theoretic Perspectives and Philosophical Framework. Dissertation (PhD in Mathematics), Universidad Nacional de Colombia. Available at: https://repositorio. unal.edu.co/bitstream/handle/unal/63339/Thesisfinal. pdf? sequence=1\&isAllowed=y.

[4] Arias, J. 2018. Spaces of Gestures are Function Spaces. Journal of Mathematics and Music, 12/2, pp. 89-105. Available at: https://doi.org/10.1080/17459737.2018.1496489.

[5] Arias-Valero, J.; Agustín-Aquino, O.; Lluis-Puebla, E. 2020. On First-Species Counterpoint Theory. Preprint, Available at: https://arxiv.org/pdf/2004.07983.pdf.

[6] Arias-Valero, J.; Lluis-Puebla, E. 2020. Explicit Presentations of Topological Categories of Gestures. Preprint, Available at: https://jusearva.files.wordpress.com/2021/04/ prestopcat-name.pdf.

[7] Arias-Valero, J.; Lluis-Puebla, E. 2020. Simplicial Sets and Gestures: Mathematical Music Theory, Infinity-Categories, Homotopy, and Homology. Preprint, Available at: https:// jusearva.files.wordpress. com/2020/08/simpgest.pdf.

[8] Arias-Valero, J.; Lluis-Puebla, E. 2020. Some Remarks on Hypergestural Homology of Spaces and Its Relation to Classical Homology. Journal of Mathematics and Music, 14/3, pp. 245-265. Available at: https://doi.org/10.1080/17459737.2020.1722269.

[9] Artin, M.; Grothendieck, A.; Verdier, J-L. 1972. Théorie des Topos et Cohomologie Étale des Schémas (SGA 4), Tome 1. Berlin: Springer-Verlag.

[10] Cadoz, C.; Wanderley, M. 2000. Gesture-Music. In: Wanderley, M.; Battier, M. (eds.). Trends in Gestural Control of Music. Ircam-Centre Pompidou. Available at: https://hal. archives-ouvertes.fr/hal-01105543.

[11] de Saint-Victor, H. 1854. De Institutione Novitiorum. In: Migne, J-P. (ed.). Patrologia Latina, Tomus CLXXVI, Hugonis de S. Victore ... opera omnia, V. 2, Cols. 925-952. Paris. Available at: https://books. google.com.co/books?id=rMQUAAAAQAAJ.

[12] Dieudonné, J. 1989. A History of Algebraic and Differential Topology 1900-1960. Boston: Birkhäuser.

[13] Godøy, R.; Leman, M. (eds.). 2010. Musical Gestures: Sound, Movement, and Meaning. Taylor \& Francis.

[14] Goerss, P.; Jardine, J. 1999. Simplicial Homotopy Theory. Basel: Birkhäuser Verlag.

[15] Grothendieck, A. 1957. Sur quelques points d'algèbre homologique. Tôhoku Mathematical Journal, 9/2, pp. 119-221.

[16] Johnstone, P. 1982. Stone Spaces. Cambridge: Cambridge University Press.

[17] Mac Lane, S. 1998. Categories for the Working Mathematician. New York: Springer-Verlag.

[18] Mac Lane, S.; Moerdijk, I. 1992. Sheaves in Geometry and Logic. New York: Springer-Verlag.

[19] Mannone, M. 2018. Knots, Music and DNA. Journal of Creative Music Systems, 2/2.

[20] Mannone, M.; Turchet, L. 2019. Shall We (Math and) Dance? In: Mathematics and Computation in Music. Cham: Springer, pp. 84-97. 
[21] Mazur, B. 2004. What Is a Motive? Notices of the AMS, 51/10, pp. 1214-1216.

[22] Mazzola, G.; Cherlin, P.; Rissi, M.; Kennedy, N. 2008. Flow, Gesture, and Spaces in Free Jazz: Towards a Theory of Collaboration. Springer-Verlag Berlin Heidelberg.

[23] Mazzola, G. 2007. La vérité du beau dans la musique. Paris: Editions Delatour France.

[24] Mazzola, G. 2009. Categorical Gestures, the Diamond Conjecture, Lewin's Question, and the Hammerklavier Sonata. Journal of Mathematics and Music, 3/1, pp. 31-58. Available at: https://doi.org/10.1080/17459730902910480.

[25] Mazzola, G. 2012. Singular Homology on Hypergestures. Journal of Mathematics and Music, 6/1, pp. 49-60. Available at: https://doi.org/10.1080/17459737.2012.680311.

[26] Mazzola, G.; Andreatta, M. 2006. From a Categorical Point of View: K-nets as Limit Denotators. Perspectives of New Music, 44/1, pp. 88-113.

[27] Mazzola, G.; Andreatta, M. 2007. Diagrams, Gestures and Formulae in Music. Journal of Mathematics and Music, 1/1, pp. 23-46. Available at: http://dx.doi.org/10.1080/ 17459730601137716.

[28] Mazzola, G. et al. 2002. The Topos of Music: Geometric Logic of Concepts, Theory, and Performance. Basel: Birkhäuser.

[29] Mazzola, G. et al. 2018. The Topos of Music III: Gestures. Cham: Springer.

[30] Merleau-Ponty, M.; Lefort, C. (ed.); Lingis, A. (trans.). 1968. The Visible and the Invisible. Evanston: Northwestern University Press.

[31] Milnor, J. 1957. The Geometric Realization of a Semi-Simplicial Complex. Annals of Mathematics, 65/2, pp. 357-362. Available at: http://www. jstor.org/stable/1969967.

[32] Mozart, W.; Broder, N. (ed.). 1960. Sonatas and Fantasies for the Piano. Bryn Mawr, PA: Theodore Presser Company.

[33] Peirce, C.; Buchler, J. (ed.). 1940. Philosophical Writings of Peirce. New York: Dover.

[34] Poincaré, H. 1895. Analysis Situs. Journal de L'École Polythechnique, 1, pp. 1-121.

[35] Schmitt, J-C. 1990. La Raison des gestes dans l'occident médiéval. Paris: Gallimard.

[36] Zalamea, F. 2012. Peirce's Logic of Continuity: A Conceptual and Mathematical Approach. Boston: Docent Press.

[37] Zalamea, F. 2017. Mazzola, Galois, Peirce, Riemann, and Merleau-Ponty: A Triadic, Spatial Framework for Gesture Theory. In: The Musical-Mathematical Mind: Patterns and Transformations. Cham: Springer, pp. 339-345. 